\title{
Powder Mixed Micro Electro Discharge Machining of Aluminium Nitride Ceramic
}

\author{
A. Bilal ${ }^{1}$, A. Rashid ${ }^{2}$, C. Liư ${ }^{2}$, M. P. Jahan ${ }^{2}$, D.Talamona ${ }^{1}$ and A. Perveen ${ }^{1, \text { a }}$ \\ ${ }_{1}^{1}$ Department of Mechanical \& Aerospace Engineering, Nazarbayev University, Nur-Sultan, 010000 Kazakhstan \\ ${ }^{2}$ Department of Mechanical and Manufacturing Engineering, Miami University, Oxford, $\mathrm{OH} 45056$ USA
}

\begin{abstract}
Advanced ceramic materials possess superior mechanical characteristics in terms of hardness, wear resistance, fracture toughness and flexural strength. However, these materials experience machining limitations due to their hardness. Machining process of such materials requires high cutting forces and results in high tool wear. Electrodischarge machining (EDM) can be considered as an alternative machining process for advanced ceramics, since this technique is a non-contact machining process, it does not involve high cutting forces but experiences moderate tool wear. However, EDM requires materials to have certain level of electrical conductivity, therefore, non-conductive and semi-conductive ceramic materials experience challenges during machining process. Assisting Electrode Method was suggested as a solution for machining of non-conductive ceramics by EDM. In this method, conductive layer is applied on top of non-conductive ceramics and thus workpiece can be machined by EDM process using residual conductive layer. In this study, coating consisting of three layers, where silver nanoparticles were sandwiched between two layers of silver and copper on top, was used as assisting electrode to machine Aluminium Nitride (AlN) ceramics by silver nanoparticles mixed micro-EDM. Successful machining of AIN was demonstrated and blind micro hole with higher than three aspect ratio was achieved.
\end{abstract}

\section{Introduction}

The electro-discharge machining (EDM) is a process where material removal occurs through evaporation and melting of workpiece. Development of this technology created an alternative way of machining special materials with particular requirements. Non-conductive ceramics are found to be promising materials that can meet demands in different areas of industry such as biomedicine, energy, transport [1]. However, for the traditional cutting technologies, machining of those materials are found to be problematic because of their high brittleness and toughness. High cutting forces that are applied in traditional cutting technologies result in surface and sub-surface cracks when applied to brittle materials. EDM is considered as a non-traditional machining process that is also force-free and therefore can be considered as an alternative machining technology which allows machining of ceramics irrespective of their hardness [2].

However, in EDM of non-conductive material, electrical conductivity is the most crucial factor. Minimum limit of machined material's electrical conductivity is $10-2 \Omega-1 \mathrm{~cm}-1$ [3]. Ceramics can be conductive, semi-conductive and non-conductive. Although conductive ceramics can be machined by EDM, semi-conductive and non-conductive ceramics do not have enough level of electrical conductivity to be

\footnotetext{
${ }^{\mathrm{a}}$ Corresponding author: asma.perveen@nu.edu.kz
}

machined by EDM [4]. Literature suggest two ways to influence electrical conductivity level of ceramic materials. First approach is to add secondary electrically conductive phase. However this kind of reinforcement affects mechanical properties of ceramics such as hardness, flexural strength and fracture toughness [5]. Second approach is to apply Assisting Electrode Method, where conductive coating is applied on top of insulating ceramics [6]. During the first spark, molecules of dielectric oil hydrocarbons and conductive coating are decomposed because of high temperature and that allows carbon elements to bind to some ceramic elements. Second spark decomposes newly formed intrinsic conductive layer together with ceramics. Crucial aspect in maintaining $s[$ machining of non-conductive ceramics is formation of secondary intrinsic conductive layer and removal of this layer with ceramics [5][7][8]. As long as this sequence is repeated steadily, machining of nonconductive ceramics is possible. In this paper, conductive coating consisting of three layers, where silver nanoparticles were sandwiched between two layers of silver and copper on top, was proposed as assisting electrode to machine AlN ceramics by Silver nanopowder mixed micro-EDM. 


\section{Experimental details}

The machining of non-conductive AlN by micro-EDM was conducted using on machine fabricated tool. MIKROTOOLS DT-110 micro-EDM machine with RC (resistor-capacitor) type pulse generator was used for the micro-EDM process. Schematic of machining tool is provided in Figure 1.

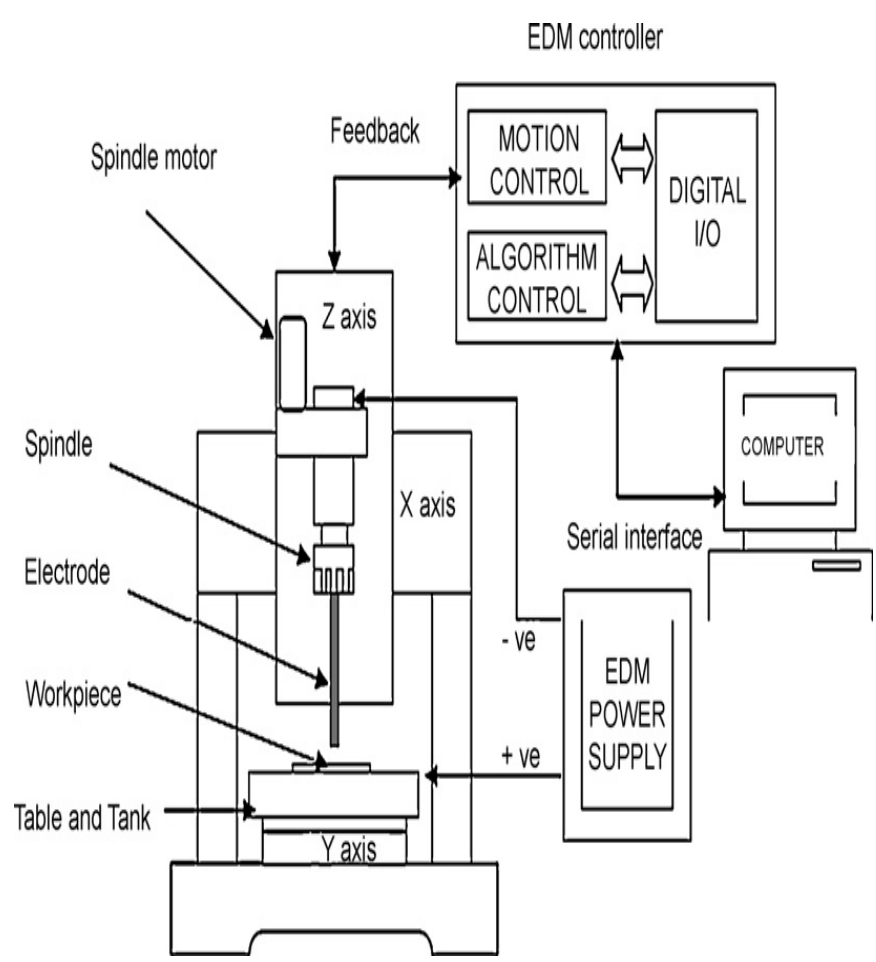

Figure 1. Schematic representation of the MIKROTOOLS DT110 micro EDM machine used in this study.

In this study, copper tungsten rods with $600 \mu \mathrm{m}$ in diameter was selected as base electrodes. The coppertungsten rods was cut to the desired length and machined to the desired diameter by block-micro EDM process. The schematic process of tungsten electrode fabrication is demonstrated in Figure 2. Copper-Tungsten tool $(\mathrm{CuW})$ with diameter of $310 \mu \mathrm{m}$ and hydrocarbon oil as dielectric along with silver nano-particles (density of 100 $\mathrm{mg} / \mathrm{L}$ ) were used. Machining was performed without tool rotation and flushing. AIN is electrically non-conductive ceramics, so conductive coating was applied on top surface of the ceramics. Coating consisted of three layers: copper top layer, silver nanoparticles middle layer and silver bottom layer. Ceramics with this coating was prepared by baking at $900{ }^{\circ} \mathrm{C}$ for an hour. The machining voltage was of $80 \mathrm{~V}$ and the capacitance used were of $100 \mathrm{nF}$ and $1000 \mathrm{pF}$. The images of machined surface of AlN ceramic workpieces were obtained by using scanning electron microscopy (SEM). Depth of machined holes were measured by optical microscope.
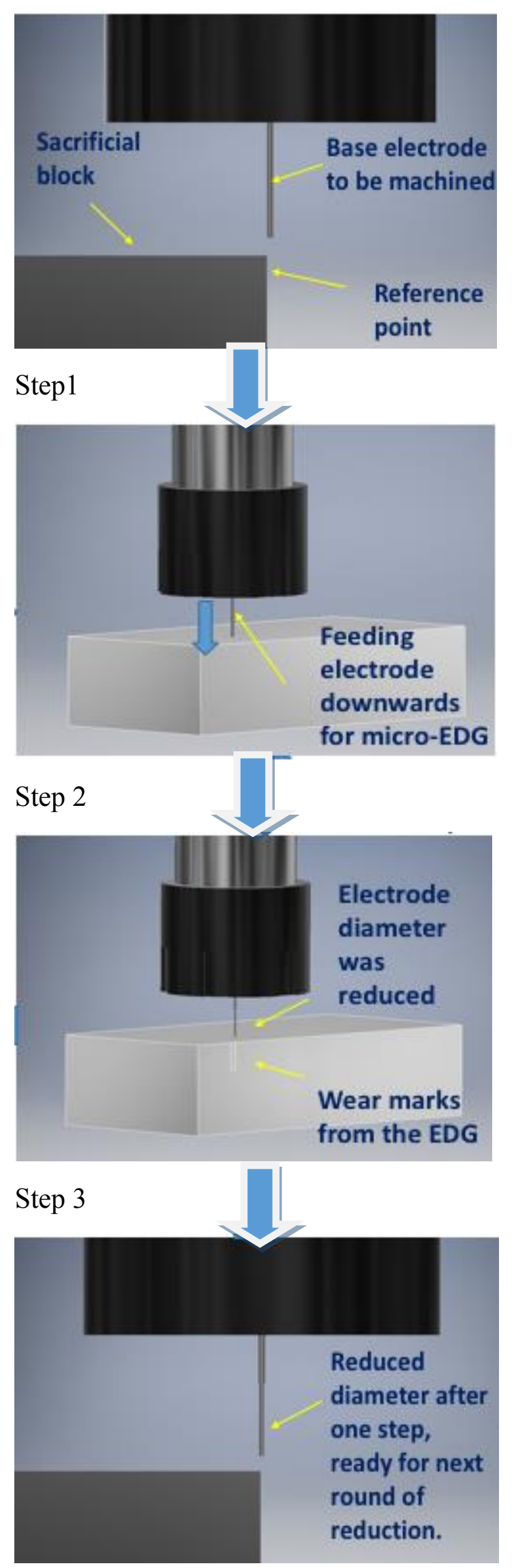

Figure 2. Steps of the microelectrode fabrication using block micro-EDG process [one cycle of reduction of diameter is shown in four step]. 


\section{Results and discussions}

SEM images of micro-EDM machined workpiece's surface are shown in Figure 3 and Figure 4 for voltage of $80 \mathrm{~V}$ and capacitance of $100 \mathrm{nF}$. These results imply that by using proposed method with three layered conductive coating, successful machining of non-conductive AlN was achieved by micro-EDM process. Depth of hole was measured by optical microscope which is $996 \mu \mathrm{m}$ measuring from coating surface.

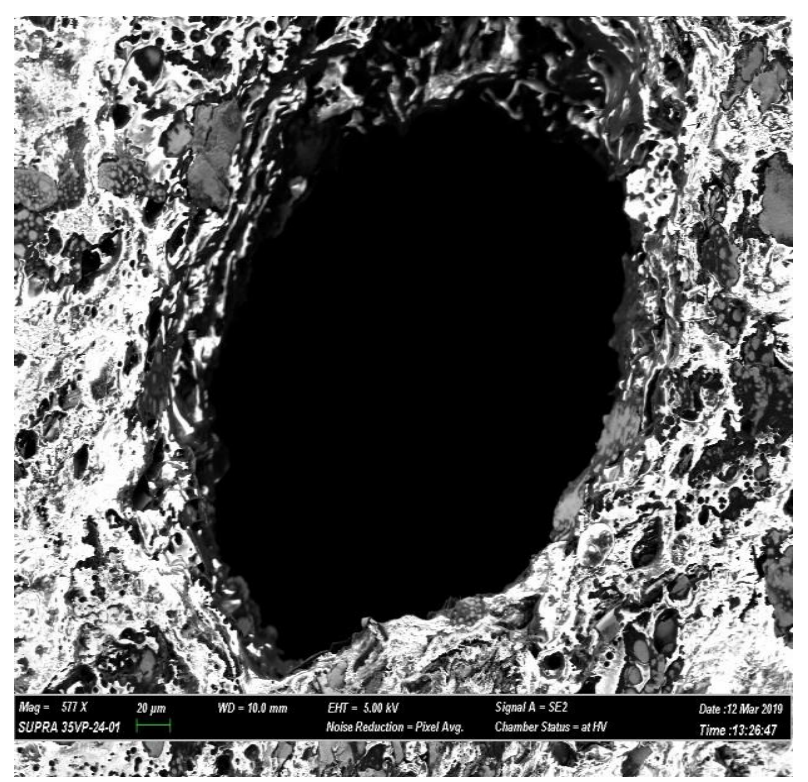

(a)

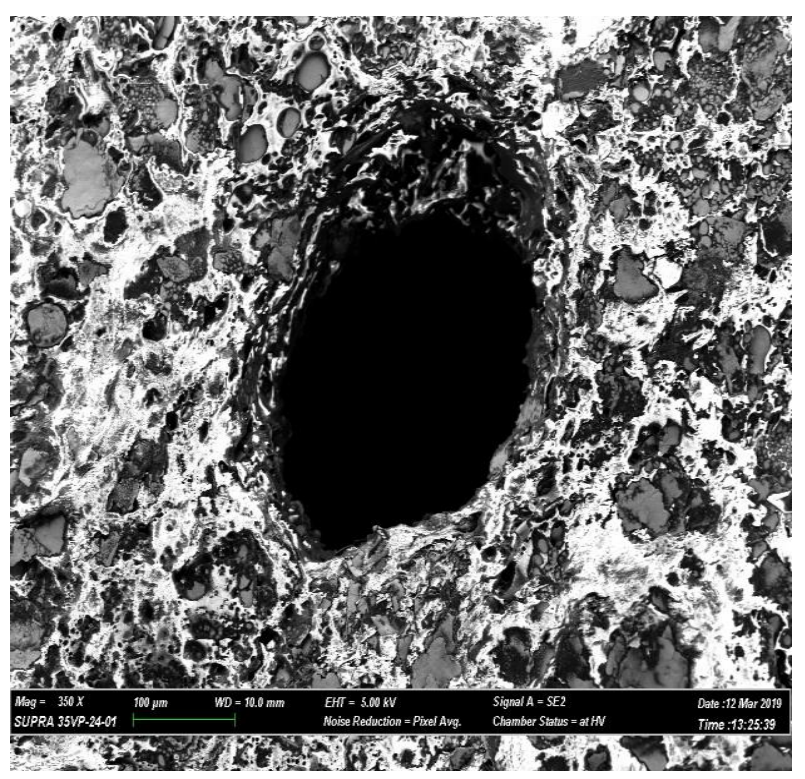

(b)

Figure $3(\mathbf{a}, \mathbf{b})$. Micro hole on coated ceramic surface (Capacitance: $100 \mathrm{nF}$, Voltage: $80 \mathrm{~V}$ ).

As a result, aspect ratio of more than 3 can be achieved using silver nanoparticles mixed EDM along with conductive coating layer. After reaching, this depth sparks were discontinuous, and electrode did not reach towards the intended depth. Figure 4 shows the side wall of the machined AIN surface. In these images, melted and foamy layer of silver on surface of AIN ceramics can be seen. Generally, the material removal mechanism of EDM is based on melting by generated plasma column due to high temperature. In Assisting Electrode Method, after the first spark occurring on the conductive coating and dielectric oil are decomposed and that results in production of carbon elements. Carbon and silver debris elements help in formation of secondary intrinsic conductive layer which maintains continuous spark generation. In addition, lack of circularity of the hole was noticed in the SEM image which might be due to the workpiece tilting at the end of the micro-EDM process and movement of the coated layer during the micro-EDM process.

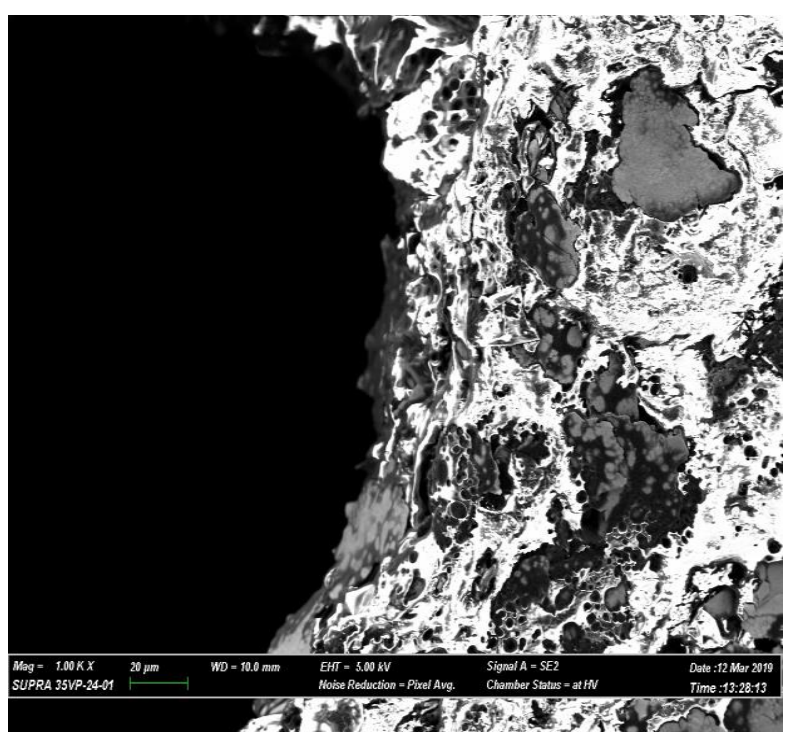

(a)

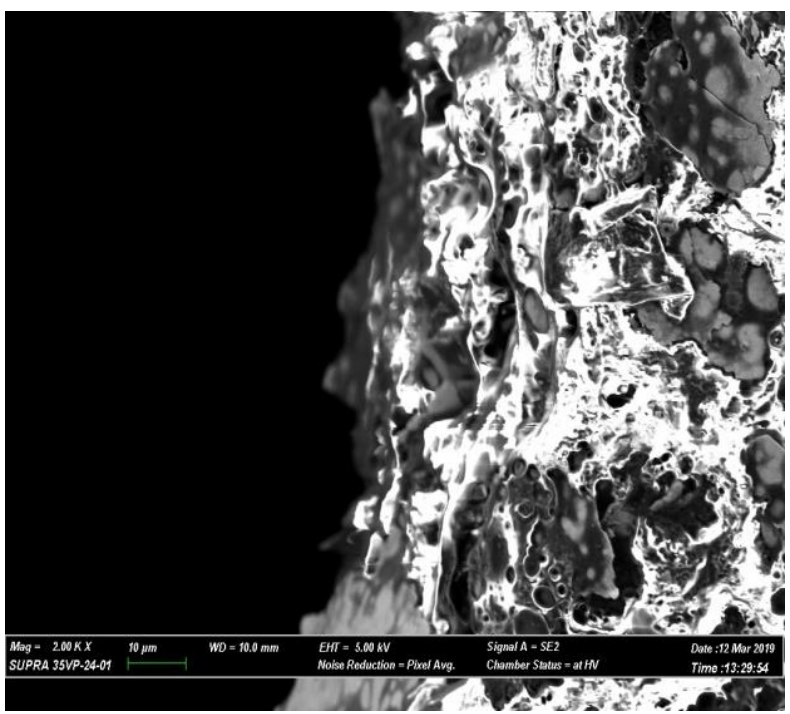

(b)

Figure 4 (a,b). Wall of the machined surface (Capacitance: 100 $\mathrm{nF}$, Voltage: $80 \mathrm{~V}$ ). 
SEM images of micro-EDM machined workpiece's surface are shown in Figure 5 for voltage of $80 \mathrm{~V}$ and capacitance of $1000 \mathrm{pF}$. Depth of hole was measured by optical microscope which is approximately $287 \mu \mathrm{m}$ (measuring from coating surface) which is much lower than the previous blind hole achieved by lower discharge energy level due to lower value of capacitance. After this depth the spark stopped, and electrode did not reach any farther down. Figure 6 shows side wall of the machined AlN surface. In this case, lack of circularity is reduced compared to the previous machining which might be due to the movement of coating layer during the EDM process.

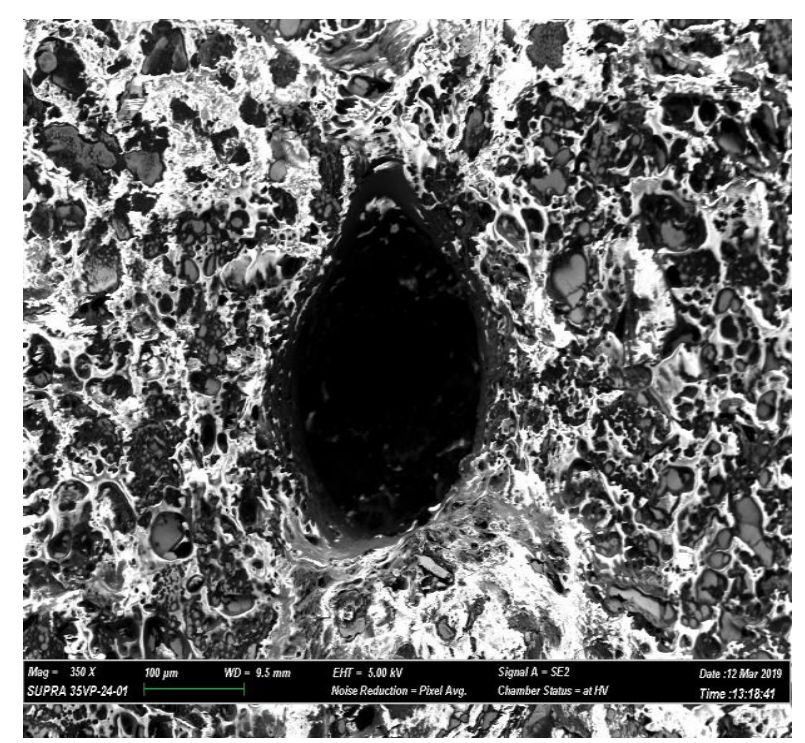

(a)

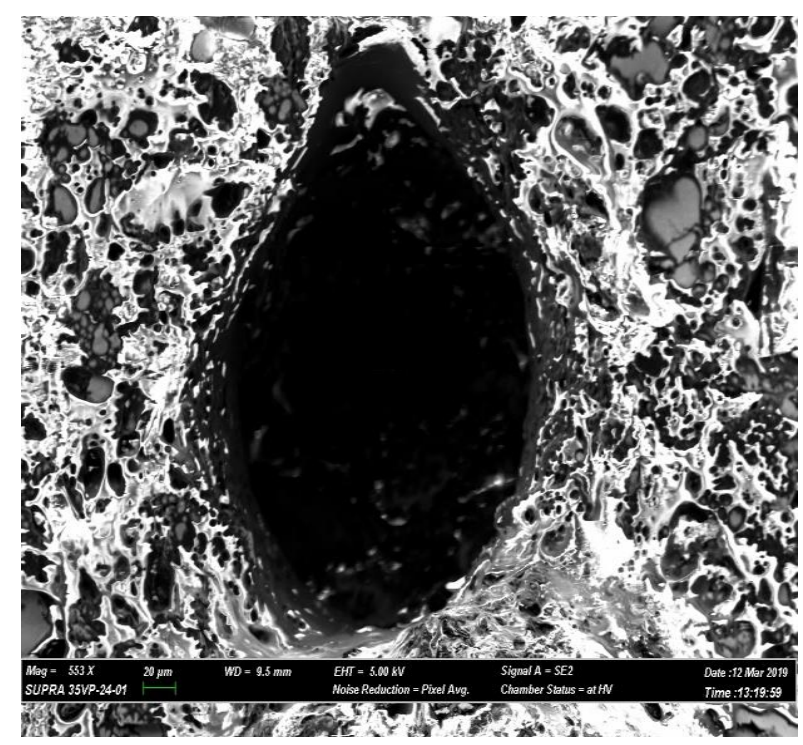

(b)

Figure $5(\mathbf{a}, \mathbf{b})$. Micro hole on coated ceramic surface (Capacitance: 1000 pF, Voltage: 80V).

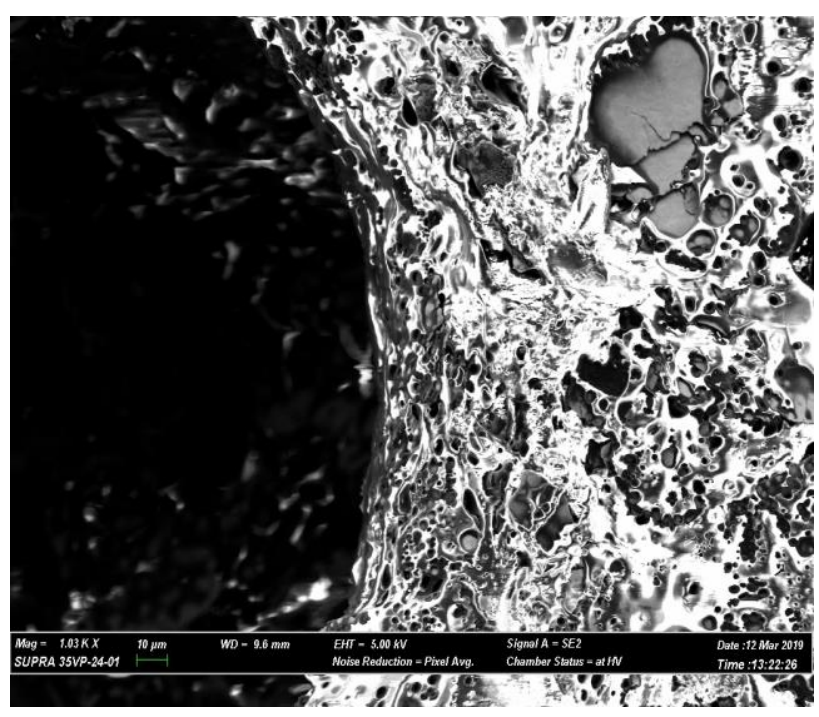

Figure 6. Wall of the machined surface (Capacitance: $1000 \mathrm{pF}$, Voltage: $80 \mathrm{~V})$.

\section{Conclusions}

This paper reported on the new improvement on sliver nanoparticles mixed micro-EDM for machining of nonconductive AlN ceramic. New conductive coating consisting of three layers, where silver nanoparticles were sandwiched between two layers of silver and copper on top, was used as assisting electrode. This method enables successful machining of non-conductive AlN where conductive particles from coating helped to maintain continuous spark generation by forming secondary conductive intrinsic layer. However, after certain depth sparking discontinues and does not reach any further down. Micro-hole with aspect ratio of 3 can be achieved using capacitance of $100 \mathrm{nF}$ and voltage of $80 \mathrm{~V}$. Further investigations may consider factors limiting maximum achievable depth and carry out optimization of process parameters for reaching intended depth.

\section{Acknowledgments}

This research study is funded by Nazarbayev University under the project "Multi-scale Investigation of the Machining Behaviour of Non-conductive Ceramics Using Electro-Discharge Machining" (grant No.: 090118FD5324).

\section{References}

1.A. Schubert, H. Zeidler, M. Hahn, M. Hackertoschätzchen, and J. Schneider, Procedia - Soc. B ehav. Sci., 6 (2013).

2. F. Ahmed, T. J. Ko, and S. Ali, Int. J. Mach. Mach. Mater., 20(1) (2018).

3. T. Hösel, C. Müller, and H. Reinecke, CIRP J. Manuf. Sci. Technol., 4 (4) (2011).

4. A. Bilal, M. Jahan, D. Talamona, and A. Perveen, Micromachines, 10(1) (2019). 
5. A. Sarsen, A. Bilal, M. R. Sankar, D. Talamona, and A. Perveen, Mater. Sci. Forum, 947(1996), (2019).

6. N. Mohri, Y. Fukuzawa, T. Tani, N. Saito, and K. Furutani, CIRP Ann. - Manuf. Technol.,45, (1) (1996).

7. Farooqui, Mohammed Naser, Patil, Nilesh G, Procedia-Manuf, 20 (2018).
8. Gadow, Rainer Landfried, Richard Kern, Frank, Proceedings of the III Advanced Ceramics and Applications Conference, (2016). 